\title{
BURIED GRAPHITE PILLARS IN SINGLE CRYSTAL CVD DIAMOND: SENSITIVITY TO ELECTRONS*
}

\author{
G. Conte ${ }^{1^{* *}}$, P. Allegrini' ${ }^{1}$ M. Pacilli' ${ }^{1}$, S. Salvatori ${ }^{2}$, D.M. Trucchi ${ }^{3}$, T. Kononenko ${ }^{4,5}$, \\ A. Bolshakov4,5, V. Ralchenko4,5, V. Konov 4,5 \\ ${ }^{1}$ University Roma Tre, Dept of Sciences, Rome, Italy \\ ${ }^{2}$ University Niccolò Cusano, Rome, Italy \\ 3Material Science Institute, Monterotondo Scalo, Italy \\ 4A.M. Prokhorov General Physics Institute, Moscow, Russia \\ 5National Research Nuclear University "MEPhI", Moscow, Russia
}

\begin{abstract}
The charge collection performance of a diamond-graphite detector is reported. Buried graphite pillars with high aspect ratio were formed inside a single crystal synthetic diamond slab by using a femtosecond IR laser with $200 \mathrm{kHz}$ of repetition rate. Grouped in two series and connected by graphite strips on the surface, eight independent $3 D$ electrodes were used to collect the charge carriers generated by energy deposited in the detector. Collimated ${ }^{\circ} \mathrm{Sr}, Y \beta$-particles were used to test the charge collection in coincidence and self-triggering mode among pillars rows using different irradiation geometries. The charge collected by one pillar row saturates at $1.40 \pm 0.02 \mathrm{fC}$ at $\pm 0.67 \mathrm{~V} / \mu \mathrm{m}$ with electrons impinging orthogonally the rows demonstrating a high charge carrier collection efficiency.
\end{abstract}

Key words: CVD Diamond, Electric contacts, Graphite pillars, 3 D sensors, $\beta$-particles

DOI: $10.21175 / \operatorname{RadJ} .2016 .01 .02$

\section{INTRODUCTION}

Solid carbon shows very different mechanical properties depending on $3 \mathrm{D}$ or $2 \mathrm{D}$ carbon-carbon bond organization. The first case leads to crystalline diamond with a very tight lattice and the highest mechanical hardness; the latter refers to graphite with ordered planes able to slide one respect the other and leading to lubricant properties. Moreover, considering the high energy needed to displace a single atom from the very compact lattice $\left(1.76 \times 10^{23} \mathrm{at} / \mathrm{cm}^{3}\right)$, diamond shows also important electronic properties for the fabrication of devices suitable to detect electromagnetic radiation from $\mathrm{UV}$ to the X-ray region [1] and for charge particles detection in high luminosity/high energy physics experiments [2]. Besides, its wide band gap energy (5.47 eV) makes this solid transparent to VIS-IR radiation also assuring very low electrical conductivity in the dark $\left(<10^{-15} \mathrm{~S} / \mathrm{cm}\right)$. High mobility (up to $2400 \mathrm{~cm}^{2} / \mathrm{Vs}$ ) and saturation velocity $\left(\sim 2 \times 10^{7} \mathrm{~cm} / \mathrm{s}\right)$ jointly with charge carriers lifetimes as high as $0.321 \mu$ s for electrons and 0.983 $\mu \mathrm{s}$ for holes have been demonstrated [3] in synthetic diamond samples deposited by CVD techniques. The very high thermal conductivity of single crystal diamond ( $22 \mathrm{~W} / \mathrm{cm} \mathrm{K}$ ), concurrently with mechanical and electronic features, ensures power dissipation, temperature stability, charge collection efficiency, high signal sensitivity, fast transient response, possibility of real-time analysis, low noise and radiation hardness for diamond based devices. All such characteristics are fundamental for radiation detectors suitable to monitor intense pulsed sources like UV lasers, synchrotron radiation beams and X-ray facilities. Detection and tracking of nuclear particles also require sensitive and fast detectors with low electronic noise, assured in this case by the small dielectric permittivity $(\sim 5.5)$ of diamond material. The other ordinate solid form of carbon, the graphite, thanks to its high electrical conductivity $\left(\sim 1.3 \times 10^{3}\right.$ $\mathrm{S} / \mathrm{cm}$ ) is suitable for the realization of bulk conductors even if good performances have been demonstrated in the form of thin layer for the fabrication also of conductive strips, array and pads in alternative to metallization $[4,5]$ and for buried contacts in $3 \mathrm{D}$ detector architectures [6,7]. The fabrication of amorphous or nano-graphite pillars and micro-channels inside the bulk diamond volume has been already introduced together with the electrical characteristics using different graphitization approaches [8-10], although only laser writing with ultra-short pulses allows local graphitization of the diamond bulk at any depth and along arbitrary $3 \mathrm{D}$ trajectory $[8,11]$. Nevertheless, power density control, graphitization rate and aspect ratio are very critical items to avoid mechanical cracks along the graphite/diamond interface where electronic active defects, with strong charge carrier recombination capability, could be generated by

\footnotetext{
* The paper was presented at the Third International Conference on Radiation and Applications in Various Fields of Research (RAD 2015), Budva, Montenegro, 2015.

${ }^{* *}$ gconte@fis.uniroma3.it
} 
thermal stress and volume expansion. Here we present a radiation detector with buried graphite pillars having a high aspect ratio, wired among them using surface graphite runners and without the need of more metallic pads. This $3 \mathrm{D}$ detector has been simulated, electrically characterized and tested with low activity radioactive sources demonstrating to be suitable for X-rays and electron spectroscopy.

\section{EXPERIMENTAL ISSUES}

\subsection{Material}

A single crystal CVD diamond produced by Elements Six, $3 \times 3 \mathrm{~mm}^{2}$ large and $0.5 \mathrm{~mm}$ thick was used in this work. The four lateral faces were lapped using common polishing techniques for further real time observation of the appearance and propagation of laser graphitized regions in the bulk of diamond. A 20x microscope objective and a CCD camera were used for this purpose.

\subsection{Graphitization and device realization}

The diamond sample was installed on a computer-controlled XYZ translation stage equipped with DC motors for precise $3 \mathrm{D}$ movement of the focal point regarding the sample surface. Local graphitization of the plate was performed with 400 $\mathrm{fs}$ pulses of VaryDisc50 laser (Dausinger+Giesen $\mathrm{Gmbh}$ ) operated at $1030 \mathrm{~nm}$ wavelength at $200 \mathrm{kHz}$ of pulse repetition rate. The laser beam was tightly focused inside the diamond plate and moved in XYZ directions to form conductive graphitic electrodes on the back surface and in the bulk. The pulse energy was varied in the $0.2-0.8 \mu \mathrm{J}$ range. Figure 1 shows a graphite pillar $20 \mu \mathrm{m}$ in diameter.

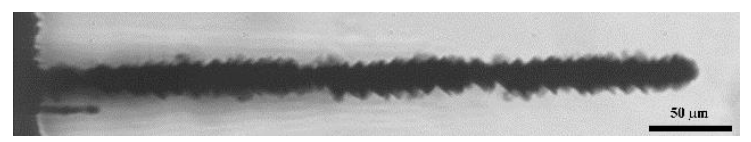

Figure 1. Graphite pillar of $20 \mu \mathrm{m}$ in diameter.

Altogether, 212 parallel buried graphite pillars, $380 \pm 20 \mu \mathrm{m}$ long (deep) and $150 \pm 5 \mu \mathrm{m}$ apart, were produced one by one, with the pillar diameter of about $20 \mu \mathrm{m}$ (see figure 1). They were grouped in two series along one direction by means of proper planar back contact patterns $(\sim 50 \mu$ m wide and $\sim 10$ $\mu \mathrm{m}$ thick, $150 \pm 5 \mu \mathrm{m}$ apart) realized with the same laser technique. The resulting contacts constitute the terminals for eight different strips (named channels in the following) used for signal acquisition surrounded by others connected pillars used for channel biasing. Due to the pillars planar body centered structure, also considering the wideness of each graphite strip and pillars diameter, in the following we will consider an effective distance among surface strips equal to Leff $=120 \pm 5 \mu \mathrm{m}$ and $\sqrt{2} \mathrm{Leff}_{\mathrm{m}} \mu \mathrm{m}$ between a couple of isolated pillars connected to different voltages.

The Raman spectra taken at the strip surface and pillar termination, on the external surface, is a proof of nano-graphite formation. The observed features in the spectra (D and G peaks) were similar to those reported elsewhere for diamond irradiated with a Ti:sapphire laser ( $1 \mathrm{ps}, \lambda=800 \mathrm{~nm}$ ). The fabricated device was then glued with silver paste on a double face printed circuit board (PCB) and the first series of pillars wire bonded with $25 \mu \mathrm{m} \mathrm{Al} / \mathrm{Si}$ standard wires directly on the graphite strips and used as powered electrode. As the formed nano-graphite shows very good adhesion to the underneath diamond, there was the possibility to directly bond the wires. This electrode is located all-around the residual series of connected pillars. The second series forms eight groups of vertical electrodes, $300 \mu \mathrm{m}$ apart, wire bonded separately - without any metallization - to copper strips on the PCB (see figure 2) which are connected to the signal input of a CAEN A1422H-F2 (45 mV/MeV in $\mathrm{Si}$ ) hybrid charge sensitive amplifier having an input test $(\mathrm{C}=1 \mathrm{pF})$ to be used for calibration.

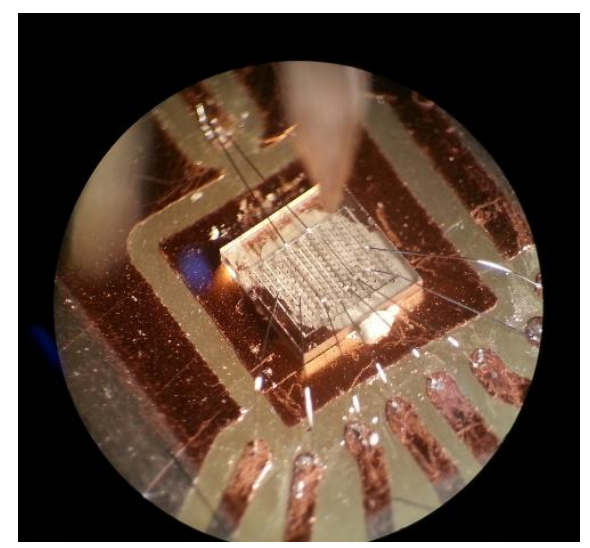

Figure 2. 8-Channels wire bonded 3D detector.

One meter long cables with LEMO and MCX connectors were used to bring signals to the digitizer. To maintain the electronic noise as low as possible, the bonding length was maintained similar and solid state batteries were used for $\pm 12 \mathrm{~V}$ low voltage amplifiers power supply. A dc-coupled configuration for supplying detector bias was also chosen to this aim being the extrinsic contribution to the noise mainly due to the detector capacitance. We have estimated the capacitance of the collection volume of a single channel, although be difficult to define its real active area. A capacitance of $0.03 \mathrm{pF}$ was calculated on the basis of 11 graphite pillars faced to 26 ones all around. This value could appear quite low and must be considered a rough lower limit estimation of a channel capacitance. On these basis, the signal-to-noise ratio of acquired curves will be defined by the observation time, digitizer voltage threshold and measuring statistics. 


\subsection{Measuring methods}

The pulses amplitude distribution and charge carriers collection of each the eight pillars group (a channel) was analyzed under collimated $1 \mathrm{MBq}$ ${ }^{90} \mathrm{Sr}, \mathrm{Y}$ test source, $1 \mathrm{~mm}$ in diameter (QSA Global, code SIFB13505, item 22/2006). The source holder and collimator was a brass cylinder $25 \mathrm{~mm}$ long, suitable to host the source, with an aperture $0.4 \mathrm{~mm}$ in diameter and $5 \mathrm{~mm}$ deep on one side, located at different distance from the detector depending on the test. Two different measurement setups were used: (i) fast counting collection in self triggering mode on each digitizer channel; (ii) slow counting collection mode using the smallest selectable coincidence window, equal to $10 \mathrm{~ns}$, between two collecting channels among the eight available ones. High voltage CAEN N1471A power supply and two different digitizers: CAEN N6724A 2-cannels, 14 bit, $100 \mathrm{MS} / \mathrm{s}$ and $500 \mathrm{mV}_{\mathrm{pp}}$ of dynamic range was used for data collection and spectroscopy analysis using CAEN DPP-PHA digital pulse processing package; V1724 8-channels, 14 bit, $100 \mathrm{MS} / \mathrm{s}$ and $500 \mathrm{mV}_{\mathrm{pp}}$ for traces dumping - 10 $\mathrm{\mu s}$ of acquisition time - using the N6724A coincidence output trigger. An Agilent $33250 \mathrm{~A}$ waveform generator with suitable rise and decay built-in curves was used to produce a calibration curve to convert the pulse height distribution in the $\mathrm{ADC}$ channels to equivalent electrons and collected charge. The calibration curve was linear with slope $\mathrm{b}=2.66 \mathrm{aC} / \mathrm{ADC}$ channel and constant parameter $\mathrm{a}=16.3 \mathrm{aC}$.

\section{RESULTS AND DISCUSSION}

\subsection{Current-Voltage characteristic}

The current versus voltage I(V) curve, in the dark, up to $\pm 100 \mathrm{~V}$ of channel-n.8 is reported in figure 3. A Keithley 6517A picoammeter was used for this purpose at room temperature and with zero current correction. Before the measurement, aimed to remove possible adsorbed water from the surface, the device was heated at $250^{\circ} \mathrm{C}$ in air for $300 \mathrm{~s}$.

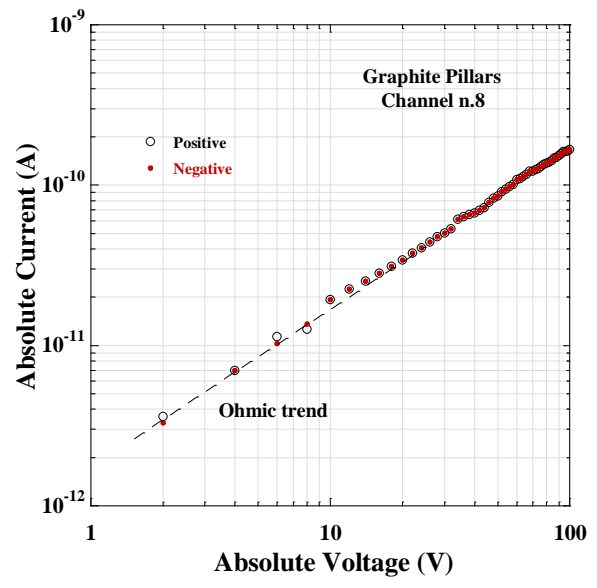

Figure 3. Channel n.8 Current vs Voltage I(V) characteristic.

The characteristic is symmetric reversing the voltage polarity and shows an Ohmic trend. In the explored voltage range, the resistance of probed volume corresponds to about $0.5 \mathrm{~T} \Omega$. The $\mathrm{I}(\mathrm{V})$ characteristics of other channels show the same features. This result evidences as none modification of force lines distribution takes place increasing the applied voltage. In other words, changing the applied voltage the electric field distribution remains constant at different depth in the bulk diamond. Indeed, at lower voltages, the conduction takes place mainly on the diamond surface between the graphitic strips connecting the pillars, $150 \mu \mathrm{m}$ apart. Inside the bulk diamond, due to the body centered structure, two pillars biased at different voltage experience a higher electric field also if their relative distance is $\sqrt{ } 2$ times the distance between surface strips. As the voltage increases, the electric field force lines penetrate deeply into the bulk diamond contributing to the charge collection process. The electric field distribution is strongly asymmetric inside the bulk diamond and around a pillar as demonstrates figure 4 where a zoom of a deep region between biased and grounded pillars is shown. The simulation has been carried out using FlexPDE 5.0.4, a finite element software package, on a structure reproducing the real fabricated device with proper material conductivity, dielectric permittivity, graphite strips on the surface and pillars immersed in the bulk diamond. 


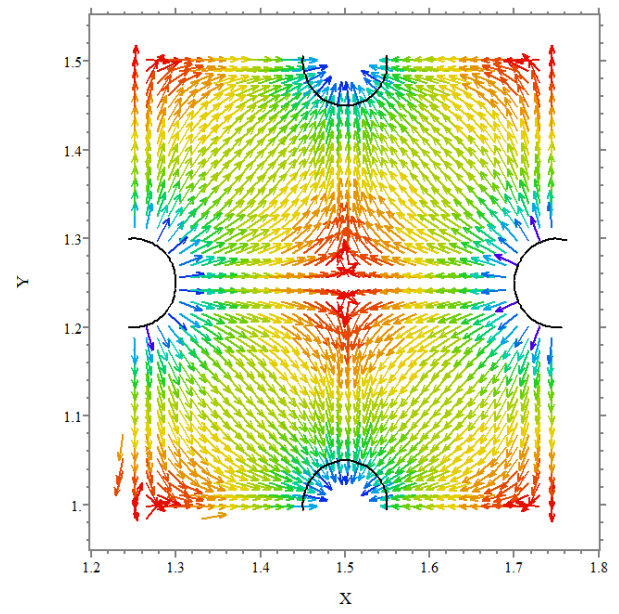

Figure 4. Electric field 3D analysis of a buried graphite pillars detector. The picture shows a zoomed region deep inside the bulk diamond. Blue color indicates high electric field.

\subsection{Response to ${ }^{\circ 0} \mathrm{Sr}, Y \beta$-particles}

Initially, aimed to evaluate the eight channels collection features, the signal dependence on voltage was measured in self-triggering mode on all channels at parallel (vertical) incidence with respect to the pillars row showing their homogeneous response at constant voltage with the source aligned to the channel.

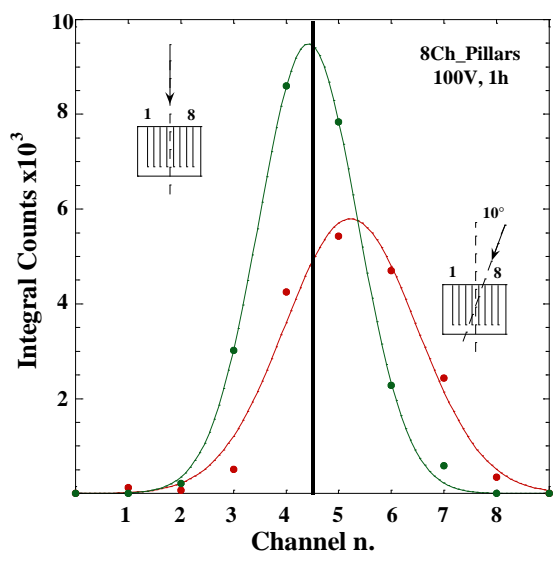

Figure 5. Integral number of events measured by the eight channels, monitored two at a time with the source aligned as indicates the inset.

With the source $1 \mathrm{~cm}$ apart the detector surface and aligned between $\mathrm{Ch}_{4}$ and $\mathrm{Ch}_{5}$, the integral number of events (green curve) acquired by each channel is reported in figure 5 . The number of events follows a Gaussian distribution centered between $\mathrm{Ch} 4$ and Ch5. Two more dummy channels, having no counts, have been added. In the same figure it is also reported the integral number of events with an impinging angle of $10^{\circ}$ with respect the vertical direction (red curve). It is worth to note that, as the distribution changes, the maximum decreases in value and shifts to channel position 5.3 whereas the FWHM increases towards an equivalent value of three channels. This result addresses the possibility to use this detector for angle resolved measurements.

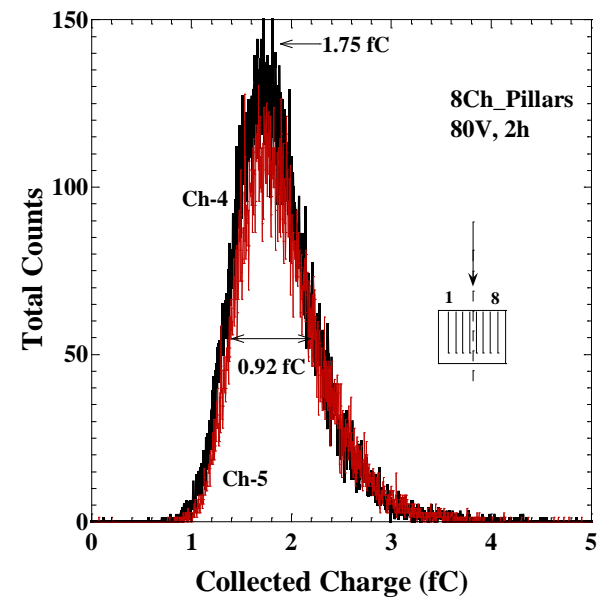

Figure 6. Pulse height distribution of central $\mathrm{Ch}_{4}$ and $\mathrm{Ch}_{5}$ at $80 \mathrm{~V}$ with the orthogonal setup.

The pulse height distributions at $80 \mathrm{~V}$ of $\mathrm{Ch}_{4}$ and $\mathrm{Ch}_{5}$ are reported in figure 6 . Measurements were taken using the internal digitizer self triggering configuration with a voltage threshold fixed to $0.3 \mathrm{mV}$ (12 ADC Channels). The signal monitored for one hour shows an asymmetric distribution with a long exponential decay tail. For the collected charge, the two curves show a most probable value (MPV) equal to $1.75 \pm 0.02 \mathrm{fC}$ (about 11000 equivalent electrons), and a FWHM of the distribution of $0.92 \pm 0.02 \mathrm{fC}$. ${ }^{90} \mathrm{Sr}, \mathrm{Y}$ radioactive source produces electrons having a continuous energy $\beta$ - decay spectrum. ${ }^{\circ} \mathrm{Sr}$ decays by $\beta^{-}$emission to ${ }^{\circ} \mathrm{Y}$ with an end-point energy $0.53 \mathrm{MeV}$, then new $\beta^{-}$decay to ${ }^{\circ} \mathrm{Zr}$ is observed with an endpoint kinetic energy of $2.28 \mathrm{MeV}$. For electrons with $0.5 \mathrm{MeV}$ of kinetic energy, the energy loss in a $500 \mu \mathrm{m}$ diamond film is approximately $\Delta \mathrm{E} \sim 0.35 \mathrm{MeV}$. Thus, electrons with energy below $0.5 \mathrm{MeV}$ are completely stopped whereas those with kinetic energy above 1.2 $\mathrm{MeV}$ could be able to traverse all the diamond slab (i.e. $3 \mathrm{~mm}$ ) behaving like MIPs (Minimum Ionizing Particles) and producing a mean of $36 \mathrm{e}-\mathrm{h} / \mu \mathrm{m}$ [12]. We will use this number for a rough estimation of the equivalent produced electrons. The detector design with the graphite strips on the top side and the body centered pillars ending at about $100 \mu \mathrm{m}$ from the bottom side produces the strong asymmetry in the electric field force lines, already addressed in figure 4, with wide regions of low electric field strength. Moreover, this particular pillars structure also produces a non-uniform electric field in the vertical direction, completely different from the situation of a 
"classical" 2D planar capacitor-like architecture: in other words, it is reasonable expected a poor charge collection efficiency. If we consider, for the sake of simplicity, a high charge collection distance in this single crystal diamond plate, we could consider to collect the charge along the $380 \mu \mathrm{m}$ of average thickness and inside the whole volume among pillars, even if with unknown efficiency. The measured distribution MPV at $1.75 \pm 0.02 \mathrm{fC}$ and the average collected charge value $\langle\mathrm{Q}\rangle=1.85 \pm 0.02 \mathrm{fC}$ indicate a promising result for the implemented detector geometry and demonstrate once again that graphite pillars buried in single-crystal diamond are suitable $3 \mathrm{D}$ electrodes for detecting $\beta$-particles with high charge collection efficiency.

Besides this study, the response at orthogonal incidence with the source $2 \mathrm{~cm}$ apart the pillars row was tested. Figure 7 shows the signal dependence on the collected charge for positive voltages of channel n.8, the nearest to the source and about $0.4 \mu \mathrm{m}$ from the impinging face. The spectrum is mainly due to low-energy electrons penetrating no more than 0.5 $\mu \mathrm{m}$.

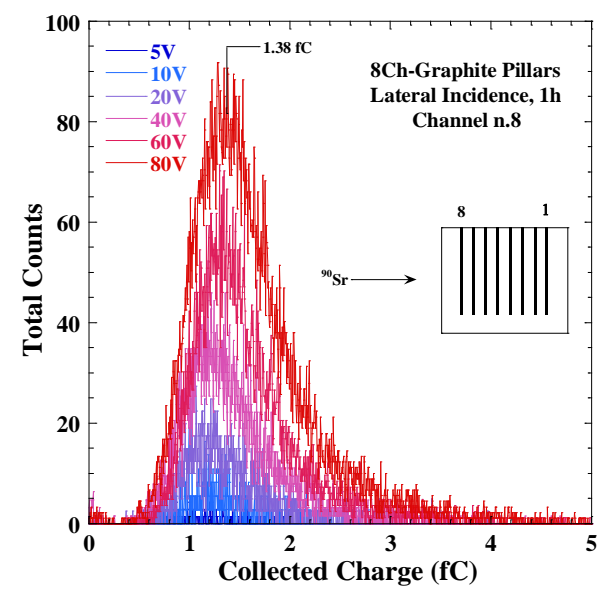

Figure 7. Channel n.8 signal dependence on positive voltage at orthogonal incidence.

The signal increases with voltage and charge collection saturation is observed at $1.38 \pm 0.02 \mathrm{fC}$ (8625e) around $80 \mathrm{~V}$ while the integral number of events increases. The signal dependence on collected charge for negative applied bias is reported in figure 8. Before performing such measurements, the detector was biased at $0.0 \mathrm{~V}$ for 24 hours in the dark. The digitizer configuration parameters, as voltage threshold, pole-zero cancellation and gain, were not changed with the exception of reversing the signal input. The distribution again saturates around $-80 \mathrm{~V}$, with a collected charge of $1.40 \pm 0.02 \mathrm{fC}$ (8750e). More parameters of curves in figure 7 and 8 are reported in Table 1 for comparison. If we assume the collection volume be defined by the effective distance between two biased pillar's rows at $250 \mu \mathrm{m}$, we should expect a release of 9000 equivalent electron charge $(\sim 1.44 \mathrm{fC})$. This estimation remains, also in this case, very rough because the body centered pillars organization does not give the possibility to exactly define the active collection volume also assuming the charge carriers diffusion.

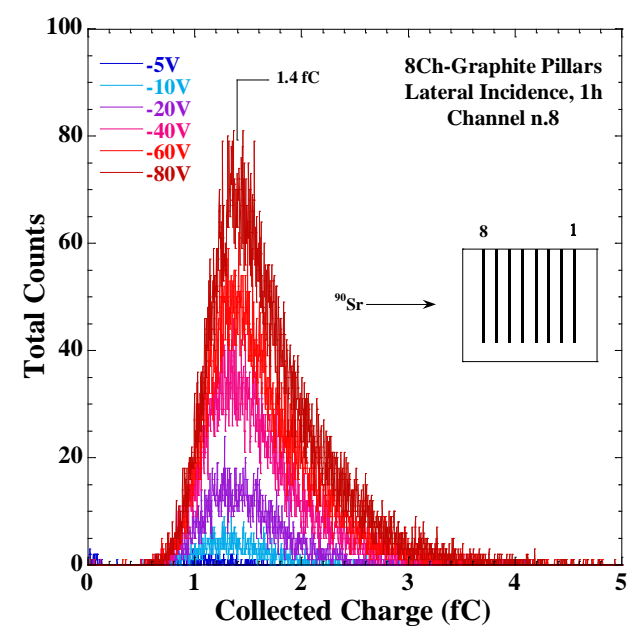

Figure 8. Channel n.8 signal dependence on negative voltage. The scheme illustrates the incidence geometry.

The small number of total measured events in one hour of acquisition, mainly due to the small collimator aperture, determines a very low signal-to-noise digital ratio; nevertheless, the average collected charge value of $1.39 \pm 0.02 \mathrm{fC}$ changing the bias polarity compares very well to the proposed estimation of $1.44 \mathrm{fC}$ and indicates once again a promising situation for this $3 \mathrm{D}$ detector and impinging particles geometry for tracking.

Table 1. Relevant parameters of Figure 7-8.

\begin{tabular}{|c|c|c|c|}
\hline $\begin{array}{c}\text { Voltage } \\
\text { (V) }\end{array}$ & $\begin{array}{c}\text { MPV } \\
\text { (fC) }\end{array}$ & $\begin{array}{c}\text { Peak } \\
\text { Counts }\end{array}$ & $\begin{array}{c}\text { FWHM } \\
\text { (fC) }\end{array}$ \\
\hline-80 & 1.40 & 70 & 1.00 \\
\hline-60 & 1.39 & 50 & 0.90 \\
\hline-40 & 1.35 & 33 & 0.83 \\
\hline-20 & 1.32 & 14 & 0.80 \\
\hline-10 & 1.25 & 4 & 0.65 \\
\hline-5 & 1.12 & 2 & - \\
\hline 5 & 1.10 & 2 & - \\
\hline 10 & 1.18 & 7 & 0.64 \\
\hline 20 & 1.16 & 15 & 0.76 \\
\hline 40 & 1.20 & 34 & 0.83 \\
\hline 60 & 1.37 & 54 & 0.90 \\
\hline 80 & 1.38 & 78 & 1.00 \\
\hline
\end{tabular}

Before experiments at beam test facility and really evaluate the detector suitability to track a single particle impinging orthogonally the pillars row, we tried to use electrons with energy greater than 1.2 $\mathrm{MeV}$, emitted by the radioactive source, for a simple tracking test. The coincidence trigger signal output from a N6724A digitizer - in coincidence mode with 10 ns of acquisition time window between Ch8 and Ch1 - 
was used to activate V1724 digitized and dump 1000 points with $10 \mathrm{~ns}$ of resolution of the residual Ch2-7 channels traces. The normalized pulse amplitude distribution measured in coincidence mode by N6724A digitizer is reported in figure 9.

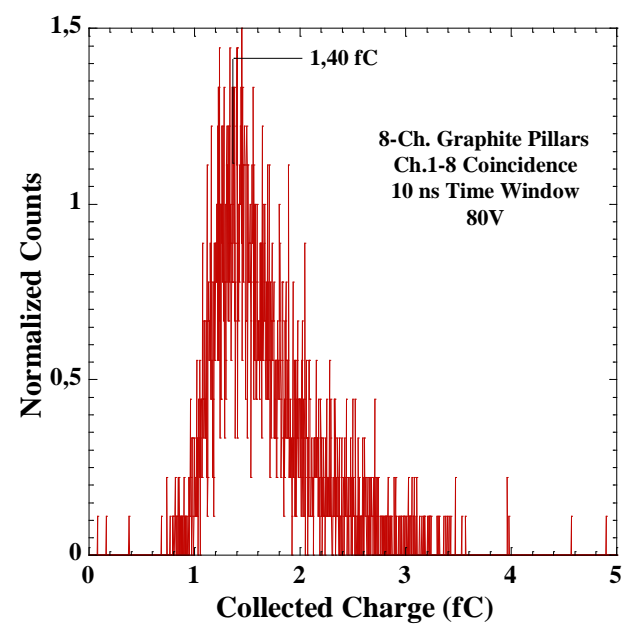

Figure 9. Coincidence pulse height distribution of $\mathrm{Ch} 8$ and Ch1.

An acquisition rate of 10 events/h has been evaluated on the two weeks of observation time. It has to be observed that the graphite pillars of Ch8 and Ch1 are about $2.1 \mathrm{~mm}$ apart. Notwithstanding the wide coincidence window, for the sake of discussion, there is the possibility to believe the spectrum associated at the energy deposited by the same electron traversing the whole distance between Ch8 and Ch1. On the basis of this hypothesis, we have to find corresponding transitions on the other channels Ch2-7 channels for each coincidence event.

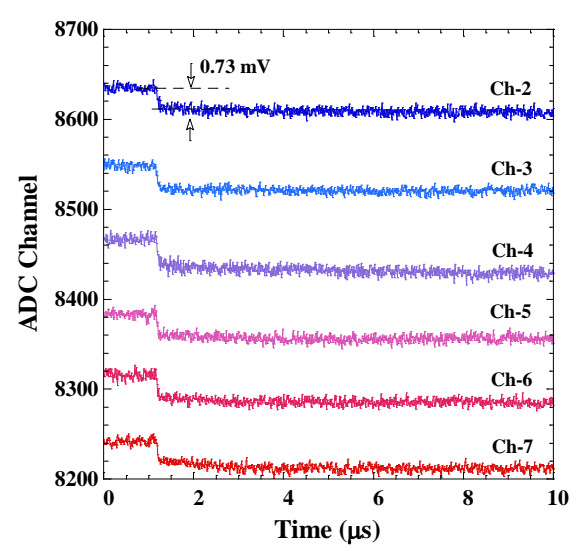

Figure 10. V1724 traces corresponding at Channel n.2 trough 7 acquired in coincidence with Channel n.1 and n.8.

For instance, figure 10 reports one of such coincidence dump related to channels 2-7 connected to the V1724 digitizer. Each of the six measured channel has a different offset for better trace visibility. As it is apparent, a step around $1 \mu$ s is visible on each channel at the same time demonstrating the coincidence among the eight graphite pillars channels. The step amplitude has been evaluated at $0.73 \pm 0.06$

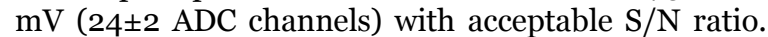
To understand the meaning of the showed step, it is worth noting that a $27.8 \mathrm{mV}$ reference pulse sent to the charge sensitive amplifier $\left(\mathrm{C}_{\text {Test }}=1 \mathrm{pF}\right)$ by the Agilent 33250A waveform generator produces a step $420 \pm 10$ ADC channel wide. Thus, showed pulse roughly corresponds to one event around $1.7 \mathrm{fC}$.

\subsection{Discussion}

The high efficiency of charge collection revealed in this study agrees with the results reported for other $3 \mathrm{D}$ diamond-based detectors fabricated by using femtosecond pulsed laser $[6,7]$. On the contrary, the detectors of similar geometry produced with nanosecond pulses [7,13] demonstrate significantly lower charge collection efficiency. The opposite tendency is found, however, for electrical conductivity of the graphitized wires: usage of nanosecond pulses provides higher conductivity by one order of magnitude comparing with femtosecond pulses (see, for instance, [14]). Raman spectroscopy is the only technique applied up to now to investigate composition and internal structure of the graphitized region in the diamond bulk. It reveals some important features like strong mechanical stresses (up to $10 \mathrm{GPa}$ ) in the surrounding diamond or intensive fluctuations of graphite concentration over the wire's length and diameter $[7,14]$, however, these findings don't explain the mentioned trends in electronic properties. It is noted [15] that full diamond-graphite transformation inside diamond bulk is impossible due to expected rise of the material compression over the limits, where such transformation is permitted by the thermodynamics. This means that the so-called "graphitized" region unambiguously contains both $s p^{2}$ and $s p^{3}$ carbon phases. However, we still have no information how the irradiation parameters influence relative concentration of both phases and their fracturing. The same relates also to the nature and generation mechanisms of electronic active defects. A further works is required to clarify those issues important for optimizing the detectors structures.

\section{CONCLUSIONS}

The tested diamond device has demonstrated to be sensitive to detect electrons impinging the $3 \mathrm{D}$ structure with different geometries. Effective charge collection has been found orthogonally the pillars rows at a $0.67 \mathrm{~V} / \mu \mathrm{m}$ electric field strength. The information earned using the radioactive source must be considered preparatory to experiments with monochromatic high energy electrons. 


\section{REFERENCES}

1. M. Girolami, P. Allegrini, G. Conte, S. Salvatori, D.M. Trucchi and V. Ralchenko, "Diamond Detectors for UV and X-Ray Source Imaging," IEEE-EDL, vol. 33, no.2, pp. 224-226, Feb. 2012.

2. W. Adams et al. (The $\mathrm{RD} 42$ Collaboration), "Radiation Hard Diamond Sensors for Future Tracking Applications," Nucl. Inst. Meth. Phys. Res., vol. A565, no.1, pp. 278-283, June 2006.

3. M. Pomorski et al., "Development of Single-Crystal CVD-Diamond Detectors for Spectroscopy and Timing," Phys. Stat. Sol. (A), vol. 203, no. 12, pp. 3152-3160, Dec. 2006.

4. M. Pacilli et al., "Beta Particles Sensitivity of an AllCarbon Detector," Nucl. Inst. Meth. Phys. Res., vol. A738, pp. 119-125, Feb. 2014.

5. A. Oh, B. Caylar, M. Pomorski and T. Wengler, "A Novel Detector with Graphitic Electrodes in CVD Diamond," Diamond Relat. Mater., vol. 38, pp. 913, 2013.

6. T. Kononenko et al., "All-Carbon Detector with Buried Graphite Pillars in CVD Diamond," Appl. Phys., vol. A114, pp. 297-300, Nov, 2013.

7. S. Lagomarsino et al., "Three-Dimensional Diamond Detectors: Charge Collection Efficiency of Graphitic Electrodes," Appl. Phys. Lett., vol. 103, pp. 23350710, Feb. 2013.

8. T.V. Kononenko,V.I. Konov,S.M. Pimenov,N.M. Ros-sukanyi, A.I. Rukovishnikov and V. Romano, "Three-Dimensional Laser Writing in Diamond Bulk," Diamond Relat. Mater., vol. 20, pp. 264-268, Jan. 2011.
9. V.P. Popov, L.N. Safronova, O.V. Naumova, D.V. Nikolaev, I.N. Kupriyanov and Yu.N. Palyanov, "Conductive Layers in Diamond Formed by Hydrogen Ion Implantation and Annealing", Nucl. Inst. Meth. Phys. Res., vol. B282, pp. 100-107, Jan. 2012.

10. F. Picollo et al., "Fabrication and Electrical Characterization of Three-Dimensional Graphitic Microchannels in Single Crystal Diamond," New Jour. Phys., vol. 14, pp. 053011-20, May 2012.

11. B. Sun, P.S. Salter and M.J. Booth, "High Conductivity Micro-Wires in Diamond Following Arbitrary Paths," Appl. Phys. Lett., vol.105, pp. 231105 (1-4), Dec. 2014.

12. S. Zhao, "Characterization of the Electrical Properties of Polycrystalline Diamond Films," Ph.D. dissertation, Dept. Phys., Ohio State Univ., Columbus, USA, 1994.

13. B. Caylar, M. Pomorski and P. Bergonzo, "LaserProcessed Three Dimensional Graphitic Electrodes for Diamond Radiation Detectors", Appl. Phys. Lett., vol.103, pp.043504 (1-3), July 2013.

14. S. Lagomarsino et al., "Electrical and Raman-Imaging Characterization of Laser-Made Electrodes for 3D Diamond Detectors", Diamond Relat. Mat., vol. 43, pp.23-28, Mar. 2014.

15. T.V. Kononenko, A.A. Khomich and V.I. Konov, "Peculiarities of Laser-Induced Material Transformation Inside Diamond Bulk," Diamond Relat. Mater., vol. 37, pp. 50-54, Aug. 2013. 